\title{
Design and Implementation of a Low-Power ZigBee Wireless Temperature Humidity Sensor Network
}

\author{
Shuipeng Gong ${ }^{1}$, Changli Zhang ${ }^{1,2}$, Lili Ma ${ }^{1}$, Junlong Fang ${ }^{1}$, and Shuwen Wang ${ }^{1}$ \\ ${ }^{1}$ Northeast Agricultural University, Harbin, Heilongjiang Province, \\ P.R. China, 150030, \\ gongshuipeng@126.com \\ ${ }^{2}$ Northeast Agricultural University, Harbin, Heilongjiang Province, P.R. China, 150030, \\ Tel.: +86-451-55190456, \\ zhangcl@neau.edu.cn
}

\begin{abstract}
The key technology of greenhouse facilities is the monitoring of environmental parameters. Now, monitoring system of greenhouse is based on wire transmission. It is complicated to route wire and difficult to maintain. Also its reliability and anti-interference performance will degrade because of heat, light and acid. This paper leads a low-power and short range ZigBee technique into greenhouse monitoring system. In order to compose intelligent network sensor system, the paper analyses the composition of network nodes power consumption, proposes low-power design method both in hardware and software. The paper selects CC2430 module which composed of transceiver and microprocessor, and use SHT15 temperature humidity sensor. After hardware and software debugging, this wireless network can acquire and transmit data of greenhouse temperature and humidity accurately and rapidly, and this system resistant to stable work, tight structure, large loads and low power consumption.
\end{abstract}

Keywords: Greenhouse, CC2430, ZigBee, Low-power, Temperature humidity sensor, Wireless network.

\section{Introduction}

Now, monitoring system of greenhouse is based on wire transmission. It is complicated to route wire and difficult to maintain. Also its reliability and antiinterference performance will degrade because of heat, light and acid. As wire transmission brings many disadvantages, more and more monitoring systems use wireless sensor networks.

ZigBee plays an important role in wireless sensor networks. ZigBee as a priority in wireless sensor networks because its advantages, such as low power consumption, high tolerance, Ad Hoc Networks [1].

In normal ZigBee network nodes use portable power (battery). Its harsh environment and large quantities make it very difficult to replace. So reducing the power consumption and extending the reliable working hours of sensor nodes become one of the key considerations. To compose intelligent sensor network system, the paper analyzes the consumption of the network and proposed low-power consumption methods both form hardware and software. 


\section{System Design}

The system uses a tree topology and consists of coordinator, router and terminal node. Each router and terminal node carries temperature humidity sensor. The coordinator connects PC by RS-232 serial ports. It collects all nodes' information and sends them to $\mathrm{PC}$ so that the manager can monitor and manage the information. Figure 3 shows the framework of the monitor system.

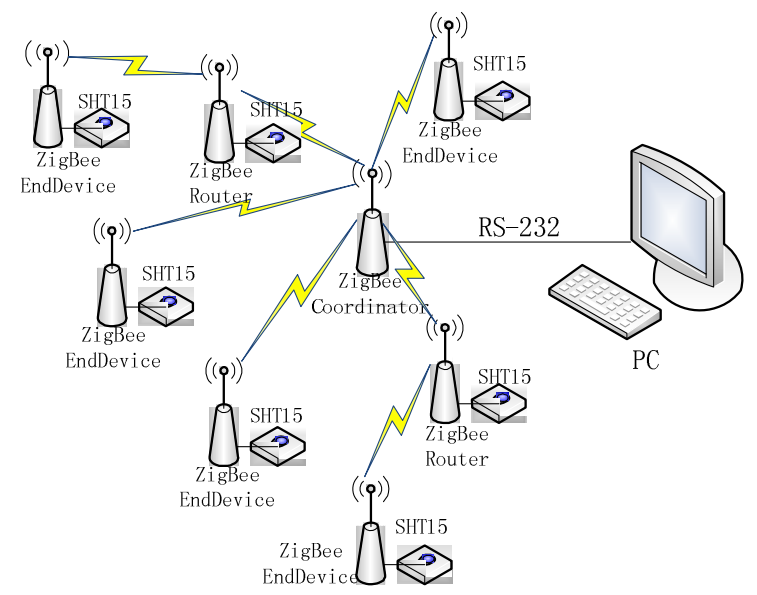

Fig. 1. The system uses a tree topology. This shows the framework diagram of the monitor system.

\section{Low-Power Hardware Designs}

The hardware of the entire network includes coordinator and sensor nodes. Coordinator is powered by USB as coordinator connects with PC. So the low power strategy of the system mainly use in ZigBee nodes. ZigBee nodes are composed of processor module, wireless communication module, temperature humidity sensor module and power module. Figure 2 shows the structure of node.

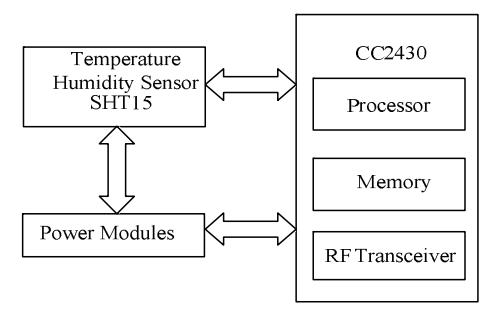

Fig. 2. The node is composed of CC2430, SHT15 and power modules 


\subsection{Processor Module}

Microprocessor is the central processing unit of the node. As the data that the microprocessor handled is large, the microprocessor is one of the most energy consuming components of the node. In the low power design, the microprocessor that the node used should be low power consumption and support sleep mode. As wireless communication account for most of the power consumption, the selection of the wireless module is very important. The following advantages of CC2430 make it become the ideal solution to solve the problem. CC2430 SoC integrates the CC2420 $\mathrm{RF}$ transceiver and enhanced 8051MCU. Its current consumption is $0.9 \mathrm{uA}$ on sleep mode and can be waked up by external interrupt or RTC wake-up system. Its current consumption is $0.6 \mathrm{uA}$ on dormancy mode and can be waked up by external interrupt. CCC2430 requires a large voltage supply between $2.0 \mathrm{~V}$ and $3.6 \mathrm{~V}$ [2].

\subsection{Sensor Module}

Sensor module uses temperature humidity sensor module SHT15. SHT15 integrates temperature humidity sensor, the conditioning and amplifying, A/D converting and $\mathrm{I}^{2} \mathrm{C}$ bus in one chip.

The serial interface of SHT15 has a definite advantage both on the reading of sensor signor and power consumption. Current consumption is $550 \mathrm{uA}$ in measuring, $28 \mathrm{uA}$ in average, $3 \mathrm{uA}$ during sleep.

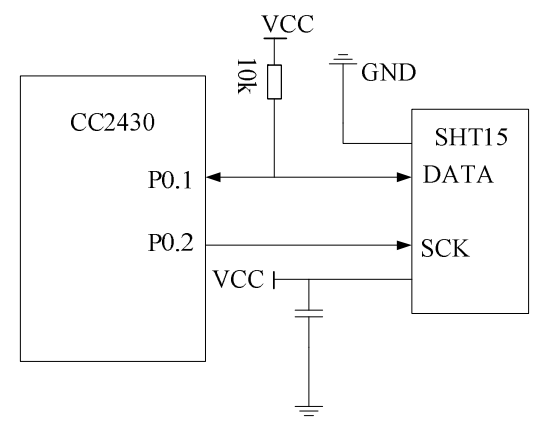

Fig. 3. This circuit diagram shows the interface of SHT15 and CC2430

\subsection{Power Module}

Power module is mainly for CC2430 and SHT15. Voltage range of CC2430 is 2.0$3.6 \mathrm{~V}$; the SHT15 is $2.4-5.5 \mathrm{~V}$. The power of the system uses $2 \# 5$ battery ( $3 \mathrm{~V})$, we can get $3.3 \mathrm{~V}$ for the system after using DC-DC L6920 step-up chip. The following advantages make L6920 become the best choice: output voltage is $3.3 \mathrm{~V}$ or $5 \mathrm{~V}$, even when the input voltage as low as $0.7 \mathrm{~V}$ the system still can work (This can make sure that after a period of time of battery usage and the input voltage is falling the output voltage is still $3.3 \mathrm{~V}$ ). This can enhance the stability of the system. The power consumption of L6920 is low. The working current is $18 \mathrm{uA}$ and only $5 \mathrm{uA}$ on sleep mode. Figure 4 shows Power circuit. 


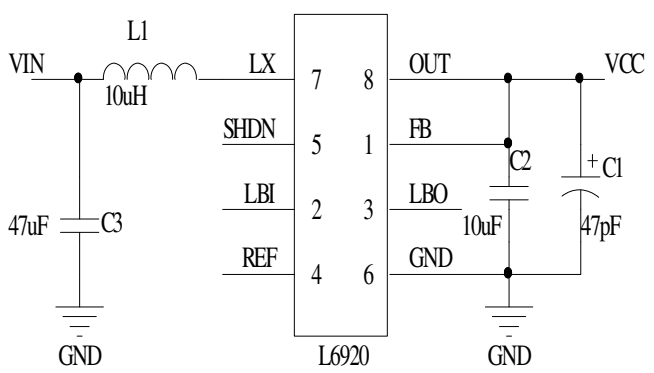

Fig. 4. Power circuit diagram shows that the $3.3 \mathrm{~V}$ battery

\subsection{Processing of Spare Pins}

The system uses the CMOS chips. The charges cumulated by spare pins make the potential between " 0 " and " 1 ". The current complementary consist of an enhanced NMOS and an enhanced PMOS, the jumping of the potential from " 0 " to " 1 " or " 1 " to " 0 " will make the two pins instant short [3]. The intermittent short will bring short consumption. So connecting spare pins to the ground can reduce power consumption.

\section{Low-Power Consumption of Software}

With the integrated circuit technological progress, power consumption of processor module and sensor module become very low. Figure 5 can explain the sensor node energy consumption, most of the energy consumption on wireless communication module; communication module has four states: transmitting, receiving, leisure and sleep, and energy consumption on transmitting, receiving and idle state is large, but the energy consumption on sleeping state is small [4]. So how to reduce power consumption on wireless communication has become an important problem.

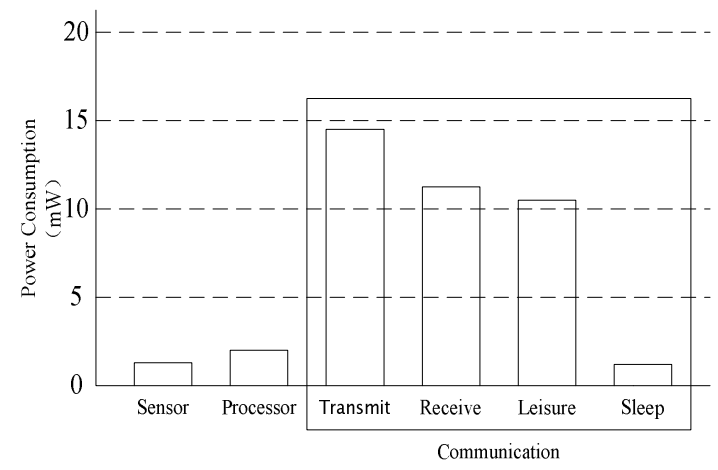

Fig. 5. This shows all the consumption of sensor node

\subsection{Working Mode of CC2430}

In general $\mathrm{CC} 2430$ has low power consumption, it offers four power modes to choose: PM0/ PM1 /PM2/ PM3. 
CC2430 works on full-function PM0 mode, the frequency is $32 \mathrm{MHz}$ and 32.768 $\mathrm{kHz}$, and the power consumption is less than $1 \mathrm{~mW}$. When it works on sleep mode PM1/PM2, Only the $32.768 \mathrm{kHz}$ low-speed crystal run, the power consumption is less than $0.9 \mathrm{uA}$, when it works on Hibernate mode PM3, there is no crystal run, so the power consumption is less than $0.6 \mathrm{uA}$.

After sending information, the node will be run into PM2-sleep mode. Because in this mode, it can run into full-function mode through internal sleep clock wake-up, and it does not require manual operation to wake-up.

When the system works on SET_POWER_MODE (2) mode, sleep time can be set. the frequency of PM2 mode is $32.768 \mathrm{kHz}$,timer of sleep is a 24-bit counter, so the longest sleep time of system is: $\mathrm{T}=2 * 24 / 32768=512 \mathrm{~s}$, the shortest sleep time is: $\mathrm{T}=1 / 32768$ and it is about $30.5 \mathrm{us}$.

The setting function of sleep time is Set_ST_Period (uint16 sec), where sec is set according to user needs.

\subsection{Low-Power Software Design}

The system wakes up nodes at regular time. Temperature and humidity is a gradual process, so it is no need to monitor it all the time. Monitoring it at regular time not only can monitor the change of temp and humidity, but also can reduce the working time of nodes. it is not necessary for the sensor nodes to communicate with coordinator all the time, this can avoid data collision and can reduce the power consumption.

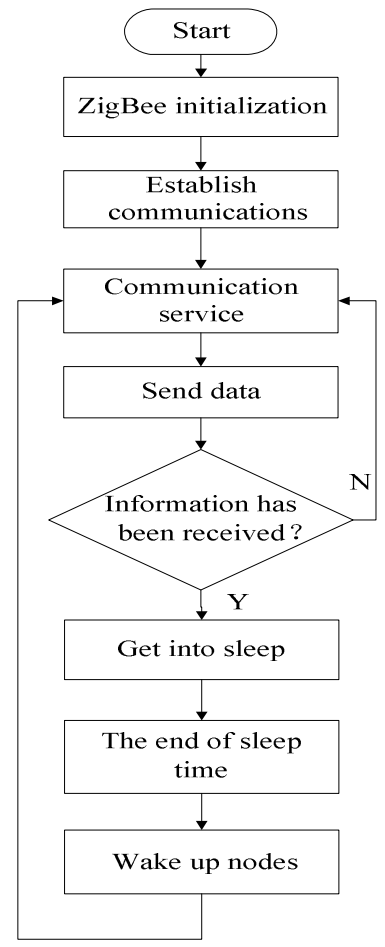

Fig. 6. This shows the flow chart of software design and the sleep mode of CC2430 
After the establishment of ZigBee networks all sensor nodes send temp and humidity information to the coordinator. After receiving the confirmed information of coordinator the node gets into sleep mode. The coordinator connects to PC through RS-232 serial port. The temp and humidity information will display in upper machine. When the wake-up time arrives, the node will rouse from sleep and continue sending messages to coordinator. This method can make all nodes in sleep state in most of the time, which will significantly reduce the power consumption of the entire network. Figure 6 shows the flow chart of software design.

\section{Implement of ZigBee Sensor Network}

The system uses a tree topology. The advantages of tree topology are as follows: it can extend transmission distance between coordinator and sensor nodes advance the ability to carry the load and enhance the area of the network.

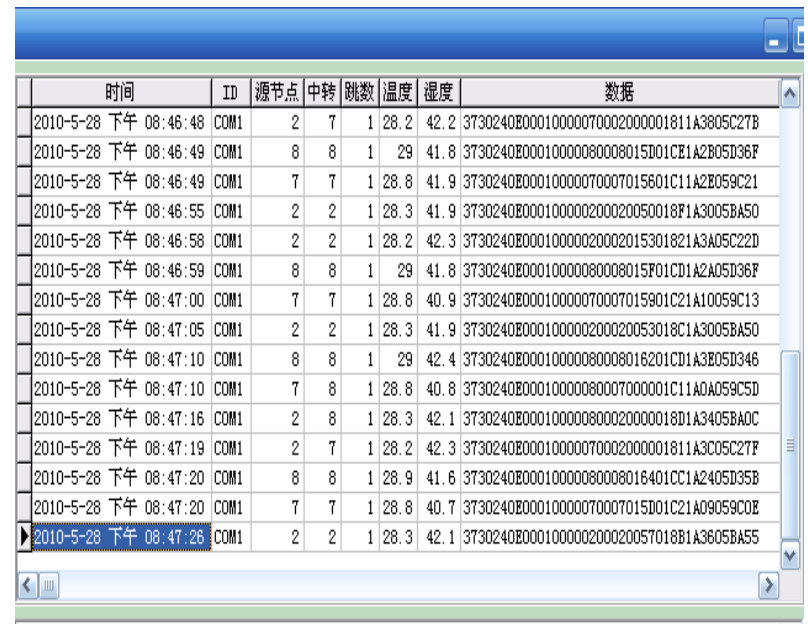

Fig. 7. This shows the results that the PC collects. The information is temperature and humidity of greenhouse.

The connection of ZigBee coordinator and sensor nodes is bonding. Bonding is a mechanism from one application layer to another [5].

$2,7,8$ are the labels of source nodes in the network, which can be determined when the program is downloaded. Each network ID is the only one in the network and each network ID correspond one sensor node [6]. We can monitor the temperature and humidity of the greenhouse through network ID. The power consumption of the nodes is $32.5 \mathrm{~mA}$ in average.

\section{Conclusion}

ZigBee wireless sensor network overcome many shortcomings that wired media bring in. This paper implements a ZigBee wireless sensor network that can transmit and 
monitor temperature and humidity of the greenhouse. This wireless sensor network resistant to stable work, tight structure, large loads and low power consumption.

After low-power design, the power consumption of the network reduces, the effective working time of the sensor nodes extend and the stability of the system enhance.

Each node can carry many sensors and controlled equipment, so the node can be added more sensor such as light sensor and $\mathrm{CO} 2$ sensor to collect more information of greenhouse.

This ZigBee wireless sensor network is part of Internet of Things, and can be used in precision agriculture and industry and many other fields.

Acknowledgments. Funding for this research was provided by Harbin Special Funds for Research on Technological Innovation Projects (NO.2008RFXXN003).

\section{References}

1. Bogena, H.R., Huisman, J.A., OberdÊrster, C., et al.: Evaluation of a low cost soil water content sensor for wireless network applications. Journal of Hydrology, 32-42 (2007)

2. Zhang, X., Zhang, C., Fang, J.: Smart Sensor Nodes for Wireless Soil Temperature Monitoring Systems in Precision Agriculture, pp. 237-240 (2009)

3. Li, W., Duan, C.: ZigBee2007/PRO experiment and practice of stack. Press of Beihang University, Beijing (2007)

4. Gao, J.: Study of energy consumption of ZigBee wireless sensor network node. Electronic Test, 1-4 (2008)

5. Sun, Y., Liu, Z.C., Cai, C.: Design of Low-power wireless sensor networks nodes. Computer Applications, 21-26 (2009)

6. Li, J., Zhang, C., Fang, J.: Design On The Monitoring System Of Physical Characteristics Of Dairy Cattle Based On Zigbee Technology. In: IEEE Proceedings of the 2009 International Conference on Computer and Computing Technology Applications in Agriculture (2010) 